\title{
RELACIÓN ENTRE LAS DIMENSIONES DEL SÍNDROME DE BURNOUT Y LOS FACTORES DE SATISFACCIÓN LABORAL
}

\section{RELATIONSHIP BETWEEN THE DIMENSIONS OF BURNOUT SYNDROME AND JOB SATISFACTION}

David García Fernández, $\mathrm{M}^{\mathrm{a}}$ del Pilar Peña Trobat, Emilio León Chaparro, Beatriz Camacho y Alicia Mateos. Universidad de Granada

El objetivo de este estudio fue analizar las relaciones existentes entre el síndrome de quemarse por el trabajo (burnout) y satisfacción laboral en el Personal de Administración y Servicios de la universidad de Granada. La muestra estuvo formada por 87 trabajadores del PAS de manera no aleatoria. El diseño del estudio fue transversal y no aleatorio. Los análisis correlaciónales mostraron una relación muy significativa entre las dimensiones del síndrome burnout y la satisfacción laboral. También se observó que estas dimensiones mantenían correlaciones específicas con cada uno de los factores de satisfacción laboral.

Palabras clave: Satisfacción laboral, burnout y dimensiones del burnout.

The purpose of this study was to examine the relationship between burnout and job satisfaction among Administration and Services Staff. The sample consisted of 87 Administration and Services Staff of Granada's University. The study was crosssectional study, and not randomized. Correlational analyzes showed a significant relationship between the dimensions of burnout syndrome and job satisfaction. It was also maintained that these dimensions specifics correlations with eat of the factors of job satisfaction.

Key word: job satisfaction, burnout \& burnout's dimensions.

Para toda empresa o servicio es vital que los trabajadores se encuentren en las mejores condiciones posibles. Esto es así, ya que el rendimiento de cualquier trabajador se ve muy influido tanto por las condiciones de su trabajo como por su estado psíquico. De tal manera, suelen rendir más en su trabajo aquellas personas que se encuentran satisfechas laboralmente. Por otro lado, el hecho de trabajar en situaciones crónicamente estresantes puede hacer aparecer problemas como el síndrome de quemarse en el trabajo o burnout, el cual implica un agotamiento emocional y una despersonalización del trabajador respecto a sus compañeros o a las personas atendidas, además de aparecer conductas negativas como absentismo o irritabilidad. Este síndrome puede dar lugar a largo plazo a la aparición de problemas de salud, como fatiga crónica, trastornos del sueño, úlceras y tensión muscular entre otros.

En España se han producido cambios importantes en las últimas décadas que han afectado al sistema universitario. Para la adaptación al Espacio Europeo de Educación Superior las enseñanzas universitarias están sometidas a cambios que son resultado, además, de las políticas de convergencia europea, de las profundas transformaciones sociales, culturales y políticas a las que estamos asistiendo, consecuencia, a su vez, de la globalización, la sociedad del conocimiento y la fuerte presencia de las tecnologías en nuestras vidas (Michevila y Calvo, 2000; UNESCO, 1998). Dichos cambios afectan a la figura del Personal de Administración y Servicios universitarios, por ejemplo el cambio de los procesos de gestión del conocimiento, mediante la introducción de las TICS que implican cambios en la política de formación y algunas 
de las funciones de todos los profesionales implicados (Secretarías, bibliotecas y conserjerías). Estos cambios, además afectan a la forma de interacción social que se establecen entre dichos profesionales y el resto de usuarios. Puede decirse que la situación actual del PAS apunta hacia un aumento de las demandas del trabajo y una perdida de control al no disponer de recursos suficientes o tener que enfrentarse a situaciones aun novedosas. Esto configura una situación idónea para la aparición de problemas asociados al estrés, como puede ser el Síndrome de Burnout.

El objetivo de este estudio no es elaborar una formula para solucionar los problemas de insatisfacción laboral, propósito realmente ambicioso y utópico. Sin embargo, se pretende llevar a cabo con la intención de encontrar cual puede ser el mejor indicador a la hora de prevenir dichos problemas. Para poder entender esto le explicaremos los dos constructos de los que partimos a continuación.

Dentro de la psicología organizacional se entiende por satisfacción laboral una actitud circunscrita al ámbito laboral, que se ha definido como el grado de afecto positivo hacia el trabajo o sus componentes (Adams y Bond, 2000), o el grado en el que a los individuos les gusta su trabajo (Price y Mueller, 1986). Locke (1976) la describió como un estado emocional agradable y positivo resultante de la valoración del trabajo personal o de las experiencias laborales.

Por otra parte, el síndrome de quemarse en el trabajo consiste en una respuesta prolongada a los estresores crónicos de naturaleza emocional e interpersonal (GrauAlberola, Gil-Monte, García-Juesas y Figueiredo-Ferraz, 2010). Este síndrome psicológico se caracteriza por síntomas de apatía y no poder dar más de sí mismo en el ámbito emocional y afectivo (agotamiento emocional), desarrollo de sentimientos y actitudes de cinismo $\mathrm{y}$, en general, de carácter negativo hacia las personas destinatarias del trabajo (despersonalización), además de la tendencia a evaluarse negativamente, de manera especial con relación a la habilidad para realizar el trabajo y para relacionarse profesionalmente con las personas a las que atienden (baja realización personal en el trabajo) (Maslach, Schaufeli y Leiter, 2001). De este modo podemos ver tres dimensiones dentro de este síndrome: agotamiento emocional, despersonalización y falta de realización profesional.

Es más que imaginable, después de la presentación de los dos constructos, que la relación entre ambos es negativa. También se puede ver claramente que la satisfacción laboral es un buen indicador del bienestar del trabajador y su buen desempeño en su puesto de trabajo. Sin embargo la relación entre satisfacción laboral y cada una de las dimensiones del síndrome de quemarse en el trabajo no son tan claras. Existen estudios donde se obtiene que la satisfacción laboral está negativamente relacionada con los niveles de agotamiento emocional $y$ despersonalización, y positivamente con la realización personal en el trabajo (Alimoglu y Donmez, 2005; Michinov, 2005). Algunos estudios concluyen que la relación más intensa se establece entre agotamiento emocional y satisfacción laboral (Doest, Maes y Gebhardt, 2006), mientras que otros obtienen que la relación más intensa se da entre realización personal en el trabajo y satisfacción laboral (Iverson, Olekalns y Erwin, 1998). Ante esta situación, este estudio pretende analizar las relaciones existentes entre la satisfacción laboral y el síndrome de quemarse por el trabajo (burnout). Como entre satisfacción laboral y burnout existe una relación significativa esperamos que esta relación se mantenga con las tres dimensiones del burnout. Sin embargo es probable que existan relaciones específicas entre los factores de la satisfacción laboral y las dimensiones del burnout. 
En primer lugar, se pretenden analizar la correlación entre satisfacción laboral y las dimensiones del burnout. De tal manera, se espera que las correlaciones entre satisfacción laboral y cada una de las dimensiones de burnout sean significativas.

Por último, pretendemos averiguar si existen correlaciones con diferentes niveles de fuerza entre factores de satisfacción laboral y dimensiones de burnout. De esta manera esperamos que existan correlaciones con niveles de significatividad diferentes entre factores de la satisfacción laboral y dimensiones del burnout. Esto resulta interesante a la hora de estudiar los factores de satisfacción laboral como predictores del burnout por sí mismos.

\section{MÉTODO}

\section{Participantes}

La muestra de sujetos que participó en el estudio estaba formada por 87 trabajadores del Personal de Administración y Servicio (PAS) provenientes de las facultades de Ciencias, Relaciones Laborales, Derecho, Medicina, Enfermería, Psicología, Ciencias de la educación, Empresariales, Filosofía y Letras de la Universidad de Granada durante curso académico 2012-2013. De los 87 participantes 37 eran hombres y 50 mujeres con una edad entre 22 y 65 años y una antigüedad en su puesto de trabajo entre 1 y 39 años.

\section{Instrumentos}

Los instrumentos usados en esta investigación fueron los siguientes:

Cuestionario de Satisfacción S20/23 (Meliá y Peiró, 1989).

Los ítems se responden con una escala de 5 puntos que valora el grado de satisfacción que el trabajador tiene con diferentes aspectos del trabajo como: satisfacción con la supervisión ( 6 ítems: relaciones con supervisor y con la supervisión recibida), satisfacción con el ambiente físico de trabajo (5 ítems: limpieza y espacio disponible), satisfacción con las prestaciones (5 ítems: salario, promoción y formación), satisfacción intrínseca (4 ítems: hacer lo que le gusta y objetivos que debe alcanzar) y satisfacción con la participación (3 ítems: capacidad para decidir y participación en decisiones del departamento). La escala permite obtener una puntuación global en satisfacción laboral resultado del promedio de las puntuaciones de los ítems que la forman. Altas puntuaciones indican mayor satisfacción laboral.

Maslach Burnout Inventory (Maslach y Jackson, 1986)

Se trata de una escala para medir el síndrome burnout. Está adaptada para muestras españolas (Gil-Monte y Peiró, 1999). Este instrumento se compone de 22 ítems y consta de tres subescalas destinadas a medir los síntomas del síndrome burnout: Realización Personal en el trabajo (8 ítems; v.g., Siento que mediante mi trabajo estoy influyendo positivamente en la vida de otros), Agotamiento emocional (9 ítems; v.g., Debido a mi trabajo me siento emocionalmente agotada/o) y Despersonalización (5 ítems; v.g., Creo que trato a algunos pacientes como si fueran objetos). Esta escala de frecuencia tiene 5 grados que van desde 1 ("Nunca") a 5 ("Diariamente"). Las puntuaciones bajas en Realización personal en el trabajo junto a altas puntuaciones en Agotamiento emocional y en Despersonalización se corresponden con altos niveles del síndrome burnout. 


\section{Diseño y Procedimiento}

Nuestra investigación se trata de un estudio transversal, en cuanto a la participación de personas de diferentes edades y con una antigüedad laboral diferente cada una. Se trata, también, de un estudio con una sola medida donde la satisfacción laboral se mide con el cuestionario de Satisfacción S20/23 (Meliá y Peiró, 1989) y el síndrome de quemarse en el trabajo o Burnout con el Inventario de Burnout de Maslach (Maslach y Jackson, 1986). Por otra parte, la selección de la muestra se realizó de manera no aleatoria y su participación en el estudio fue voluntaria y anónima. Los responsables del estudio acudieron personalmente a las distintas facultades entregando el cuestionario en mano a los sujetos que estaban presentes en el lugar de trabajo. El cuestionario incluía una introducción en la que se explicaba el procedimiento de respuesta de los ítems además de un apartado donde indicar la edad, el sexo y la antigüedad en el puesto de trabajo, pero no se presentaba información alguna de la investigación.

\section{Análisis}

Los datos se han analizado con el programa estadístico SPSS 15. Para contrastar la primera hipótesis se ha realizado una correlación entre la satisfacción laboral y las dimensiones de burnout (agotamiento emocional, despersonalización y realización personal). Para la segunda hipótesis, se ha realizado una correlación entre las dimensiones de satisfacción laboral (satisfacción con la supervisión, satisfacción con el ambiente físico de trabajo, satisfacción con las prestaciones, satisfacción intrínseca y satisfacción con la participación).

\section{RESULTADO}

En la siguiente tabla presentamos las variables con la que hemos trabajado y sus características:

Tabla 1: Estadístico descriptivos

\begin{tabular}{lccccc}
\hline & $\mathbf{N}$ & Mínimo & Máximo & Media & Desv. típ. \\
EDAD & 87 & 22 & 65 & 46.78 & 8.77 \\
SEXO & 87 & 1 & 2 & 1.57 & .49 \\
ANTIGÜEDAD & 87 & 1 & 39 & 15.47 & 8.77 \\
AGOTAMIENTO & 87 & 9 & 37 & 18.63 & 6.34 \\
EMOCIONAL & 87 & 5 & 21 & 8.56 & 3.75 \\
DESPERSONACIZACIÓN & 87 & 9 & 34 & 17.54 & 5.39 \\
DESMOTIVACIÓN & 87 & 25 & 115 & 77.46 & 17.32 \\
SASTISFACCIÓN & 87 & & & & \\
LABORAL & & & &
\end{tabular}

Para analizar los resultados hemos realizado diferentes análisis de correlaciones entre las variables del estudio (tabla 1). De dichos análisis obtenemos relaciones entre la edad, el sexo, la antigüedad en la empresa y la variable satisfacción laboral con las dimensiones de Burnout. 
Tabla 2: Análisis correlacional de las variables

\begin{tabular}{lcccc}
\hline & EDAD & SEXO & ANTIGÜEDAD & $\begin{array}{c}\text { SASTISFACCIÓN } \\
\text { LABORAL }\end{array}$ \\
SASTISFACCIÓN & -.01 & -.13 & .02 & - \\
LABORAL & & & & $-.64^{* * *}$ \\
$\begin{array}{l}\text { AGOTAMIENTO } \\
\text { EMOCIONAL }\end{array}$ & .07 & .153 & .02 & $-.31^{* *}$ \\
$\begin{array}{l}\text { DESPERSONALIZ } \\
\text { ACIÓN }\end{array}$ & .03 & -.05 & -.08 & $-.33^{* *}$ \\
DESMOTIVACIÓN & .07 & .01 & -.02 & \\
\hline
\end{tabular}

Nota: ${ }^{* *} p<.001^{* *} p<.01$

En el análisis de los resultados (Tabla 2) se puede ver que las variables edad, género y antigüedad en la empresa no mantienen correlaciones significativas ni con la variable satisfacción laboral ni con las dimensiones del síndrome burnout. En cambio, hemos obtenido que la satisfacción laboral correlaciona con el agotamiento emocional significativamente y de manera negativa $(r=-, 636 ; p<0,01)$, al igual que ocurre con la dimensión despersonalización $(r=-, 307 ; p<0,01)$, y la desmotivación personal $(r=-$ ,329; $p<0,01)$.

Para analizar la segunda hipótesis que presentamos en el estudio, se realizó otro análisis correlacional entre los cinco factores de la Satisfacción Laboral y las tres dimensiones del Síndrome Burnout, en el que obtenemos como todos los factores de satisfacción laboral correlacionan con al menos una dimensión de Burnout (véase la tabla 3).

Tabla 3: Análisis de correlación entre las variables de satisfacción personal y las dimensiones de Burnout.

\begin{tabular}{|c|c|c|c|c|c|c|}
\hline & EDAD & SEXO & ANTIGÜEDAD & $\begin{array}{l}\text { AGOTAMIENTO } \\
\text { EMOCIONAL }\end{array}$ & DESPERSONALIZACIÓN & DESMOTIVACIÓN \\
\hline $\begin{array}{l}\text { SATISFACCIÓN } \\
\text { INTRÍNSECA }\end{array}$ & .14 & -.09 & .12 & $-.49^{\star \star \star}$ & $-.21^{*}$ & $-.313^{\star *}$ \\
\hline $\begin{array}{l}\text { SATISFACIÓN CON } \\
\text { LAS } \\
\text { PRESTACIONES }\end{array}$ & .03 & -.03 & .02 & $-.45^{\star \star \star}$ & -.19 & $-.32^{\star \star}$ \\
\hline $\begin{array}{l}\text { SATISFACIÓN CON } \\
\text { EL AMBIENTE }\end{array}$ & .07 & -.17 & .12 & $-.44^{\star \star \star}$ & $-.20^{*}$ & -.18 \\
\hline $\begin{array}{l}\text { SATISFACCIÓN } \\
\text { CON LA } \\
\text { SUPERVISIÓN }\end{array}$ & -.08 & -.07 & -.11 & $-.59^{\star \star \star}$ & $-.34^{* * *}$ & $-.23^{*}$ \\
\hline $\begin{array}{l}\text { SATISFACCIÓN } \\
\text { CON LA } \\
\text { PARTICIPACIÓN }\end{array}$ & -.18 & -.15 & -.03 & $-.49^{\star * *}$ & $-.22^{*}$ & $-.30^{* *}$ \\
\hline
\end{tabular}

Nota: ${ }^{* *} p<.001{ }^{* *} p<.01{ }^{*} p<.05$

En esta tabla encontramos que la dimensión de agotamiento emocional mantiene una correlación negativa significativa con todos factores de la satisfacción laboral. La dimensión de despersonalización correlaciona significativamente y negativamente con la satisfacción de supervisión y mantiene otra correlación negativa con la satisfacción de participación. La dimensión de desmotivación muestra una correlación positiva significativa con los factores de satisfacción intrínseca, con las prestaciones y con la supervisión, además mantiene una correlación significativa con la satisfacción de supervisión. 


\section{DISCUSIÓN Y CONCLUSIÓN}

En nuestra primera hipótesis se esperaba que entre satisfacción laboral y las tres dimensiones de burnout existiesen correlaciones significativas. Por lo que se podría afirmar que la satisfacción laboral mantiene una relación con los tres tipos de dimensiones del burnout (agotamiento emocional, despersonalización y realización persona). Ante esto, podemos decir que nuestra hipótesis inicial se ha cumplido. La segunda de nuestras hipótesis pretendía encontrar si existían relaciones específicas entre los factores de satisfacción laboral y las dimensiones del burnout. En el análisis correlacional se obtuvo que las relaciones entre factores de satisfacción laboral y dimensiones de burnout fueron diferentes unas de otras, surgiendo correlaciones muy significativas, ligeramente significativas o no significativas. Este hecho hace que nuestra segunda hipótesis se confirme, lo cual nos permite afirmar la existencia de relaciones específicas entre los factores de satisfacción laboral y las dimensiones del síndrome burnout.

Según otros autores, existen unas relaciones entre satisfacción laboral y las dimensiones de burnout que son más intensas que otras. Sin embargo, los resultados de dichas investigaciones no coinciden. De este modo podemos encontrar estudios donde se establece una relación más intensa entre satisfacción laboral y realización personal (Iverson, Olekalns y Erwin, 1998) mientras otros establecen una relación más intensa de la satisfacción laboral con el agotamiento emocional y la despersonalización (Doest, Maes y Gebhardt, 2006) o, como en este caso, donde se establece una relación igual de intensa entre satisfacción y cada una de las dimensiones. Sin embargo, estas investigaciones sólo han trabajado sobre el constructo general de satisfacción laboral y no sobre los factores que lo componen, lo cual supone un nivel de análisis inapropiado o poco profundo para establecer dichas relaciones. De tal modo una persona puede puntuar como insatisfecha en determinados factores mientras se muestra satisfecha en el resto, mostrando una puntuación que no refleje dichos défits factoriales del constructo. Por lo cual a la hora de estudiar las relaciones entre la satisfacción laboral y síndrome burnout, pueden surgir casos donde haya una buena satisfacción laboral y se muestre alguna dimensión del síndrome burnout, es por eso que el nivel de análisis no sería apropiado.

El hecho de que existan diferentes grados de relación significativa nos muestra que algunos de estos factores de la satisfacción laboral podrían actuar como predictores de alguna de las dimensiones del burnout. De este modo podríamos esperar que el factor de supervisión nos indicase problemas de agotamiento emocional y/o de despersonalización, de tal manera que aquellas personas que puntúen bajo en satisfacción con la supervisión muestren problemas de agotamiento emocional o de despersonalización, o bien de los dos. Esta propuesta resulta interesante de estudiar ya que podría ser importante en el ámbito de la intervención y prevención de problemas laborales como es el síndrome de burnout, pues si los factores de satisfacción pudiesen predecir aquellos problemas derivados de las dimensiones del burnout facilitaría la identificación y el tratamiento de estos mejorando posiblemente tanto el rendimiento del empleado como su bienestar.

Debemos ser conscientes de que este estudio posee algunas limitaciones. Entre estas limitaciones encontramos que la muestra se obtenido de manera no aleatoria por lo cual es probable que no sea equilibrada por lo que no representaría bien a la población. Los datos recogidos se consiguieron mediante cuestionarios, esto hace que los datos dependan en última instancia del criterio de sinceridad de respuesta del participante. Sin embargo estas limitaciones no invalidan los resultados obtenidos en este estudio ni su comparación con otras investigaciones. 


\section{Referencias:}

Adams, A., y Bond, S. (2000). Hospital nurses job satisfaction, individual and organizational characteristics. Journal of Advanced Nursing, 32, 536-543.

Alimoglu, M.K., y Donmez, L. (2005). Daylight exposure and the other predictors of burnout among nurses in a University Hospital. International Journal of Nursing Studies, 42, 549-555

Burisch, M. (2002). A longitudinal study of burnout: The relative importance of dispositions and experiences. Work and Stress, 16, 1-17.

Doest, L., Maes, S., y Gebhardt, W.A. (2006). Personal goal facilitation through work: Implications for employee satisfaction and well-being. Applied Psychology: An International Review, 55, 192-219.

Figueiredo-Ferraz1, H., Grau-Alberola2, E., Gil-Monte1, P. R., y García-Juesas, J. A. (2012). Síndrome de quemarse por el trabajo y satisfacción laboral en profesionales de enfermería. Psicothema, 24, 271-276.

Grau-Alberola, E., Gil-Monte, P.R., García-Juesas. J.A., y Figueiredo-Ferraz, H. (2010). Incidence of burnout in Spanish nursing professionals: A longitudinal study. International Journal of Nursing Studies, 47, 1013-1020.

Iverson, D.R., Olekalns, M., y Erwin, J.P. (1998). Affectivity, organizational stressors and absenteeism: A causal model of burnout and its consequences. Journal of Vocational Behavior, 52, 1-23.

Kalliath, T.J., y Morris, R. (2002). Job satisfaction among nurses: A predictor of burnout levels. Journal of Nursing Administration, 32, 648-654.

Lee, H., Hwang, S., Kim, J., y Daly, B. (2004). Predictors of life satisfaction of Korean nurses. Journal of Advanced Nursing, 48, 632-641.

Locke, E.A. (1976). The nature and causes of job satisfaction. En M.R. Dunnette (Ed.), Handbook of industrial and organizational psychology (pp. 1297-1349). Chicago, IL: Rand McNally College Publishing Co.

Maslach, C., y Jackson, S.E. (1986). Maslach Burnout Inventory Manual. (2a ed.). Palo Alto, California: Consulting Psychologists Press.

Maslach, C., Schaufeli, W.B., y Leiter, M.P. (2001). Job burnout. Annual Review of Psychology, 52, 397-422.

Meliá, J.L., y Peiró, J.M. (1989). La medida de la satisfacción laboral en contextos organizacionales: El Cuestionario de Satisfacción S20/23. Psicologemas, 5, 59-74.

Michinov, N. (2005). Social comparison, perceived control and occupational burnout. Applied Psychology: An International Review, 54, 99-118.

Navarro, M. L. A. (2010). Esters laboral y síndrome burnout en la Universidad: Analisis descriptivo de la situación actual y revisión de las principales líneas de investigación. Anuario de psicología clínica y de la salud, 6, pp 73-78. 
Price, J.L., y Mueller, C.W. (1986). Absenteeism and turnover among hospital employees. Greenwich, CT: JAI Press.

Soler Sánchez, M.I. (2008). La evaluación de los factores de riesgo psicosocialdel trabajo en el sector hortofrutícola: el cuestionario FAPSIHOS. (Tesis doctoral). 